\title{
IDENTIFICATION OF THE DNA BINDING ELEMENT OF THE HUMAN ZNF300 PROTEIN
}

\author{
HONGLING QIU, LU XUE, LI GAO, HUANJIE SHAO, DI WANG, \\ MINGXIONG GUO and WENXIN LI*
}

State Key Laboratory of Virology, College of Life Sciences, Wuhan University, Wuhan 430072, P.R. China

\begin{abstract}
The human ZNF300 gene is a member of the KRAB/ $\mathrm{C}_{2} \mathrm{H}_{2}$ zinc finger gene family, the members of which are known to be involved in various developmental and pathological processes. Here, we show that the ZNF300 gene encodes a $68-\mathrm{kDa}$ nuclear protein that binds DNA in a sequence-specific manner. The ZNF300 DNA binding site, $\mathrm{C}(\mathrm{t} / \mathrm{a}) \mathrm{GGGGG}(\mathrm{c} / \mathrm{g}) \mathrm{G}$, was defined via a random oligonucleotide selection assay, and the DNA binding site was further confirmed by electrophoretic mobility shift assays. A potential ZNF300 binding site was found in the promoter region of the human IL-2R $\beta$ gene. The results of electrophoretic mobility shift assays indicated that ZNF300 bound to the ZNF300 binding site in the IL-2R $\beta$ promoter in vitro. Transient co-transfection assays showed that ZNF300 could activate the IL-2R $\beta$ promoter, and that the activation was abrogated by the mutation of residues in the ZNF300 binding site. Identifying the DNA binding site and characterizing the transcriptional regulation property of ZNF300 would provide critical insights into its potential as a transcriptional regulator.
\end{abstract}

Key words: ZNF300, Zinc finger, DNA binding

* Author for correspondence; e-mail: liwxlab@whu.edu.cn, tel: 86-27-68752831, fax: 86$27-68752146$

Abbreviations used: EMSA - electrophoretic mobility shift assay; IL-2R $\beta$ - IL-2 receptor beta chain; KRAB - Krüppel-associated box; TK - thymidine kinase; ZNF300 - zinc finger 300 


\section{INTRODUCTION}

Transcription factors play important roles in determining spatial and temporal gene expression patterns. They can directly regulate gene transcription by interacting with cis-regulatory DNA elements in specific genes. The $\mathrm{C}_{2} \mathrm{H}_{2}$ (Krüppel-like) zinc finger protein family is one of the largest families of mammalian transcription factors. Its members are characterized by the presence of individual or clustered $\mathrm{C}_{2} \mathrm{H}_{2}$ zinc finger motifs $\left(\mathrm{CX}_{2-4} \mathrm{CX}_{3} \mathrm{FX}_{5} \mathrm{LX}_{2} \mathrm{HX}_{3-4} \mathrm{H}\right)$, which are linked by a highly conserved $\mathrm{H} / \mathrm{C}$ linker $(\mathrm{TGE}(\mathrm{K} / \mathrm{R}) \mathrm{P}(\mathrm{Y} / \mathrm{F}) \mathrm{X})[1]$. The $\mathrm{KRAB} / \mathrm{C}_{2} \mathrm{H}_{2}$ zinc finger proteins make up approximately one third of the various zinc finger proteins found in the human genome. Many usually function as transcriptional repressors, and may play important roles in diverse developmental and pathological processes [2,3].

ZNF300 cDNA was originally cloned from a human early embryo. It was found to encode a KRAB-type zinc finger protein with twelve $\mathrm{C}_{2} \mathrm{H}_{2}$ zinc finger motifs in the C-terminus [4]. It is a nuclear protein ubiquitously expressed in most human tissues, although higher levels of expression were found in the heart, skeletal muscle and brain. There are highly conserved $\mathrm{C}_{2} \mathrm{H}_{2}$ zinc finger motifs in ZNF300, and it is absent from the mouse genome, which suggests that it may function as a transcription factor and play a unique role in higher mammals. It is of interest to determine if ZNF300 functions as a transcription factor regulating crucial genes involved in important biological processes.

As the first step in the identification of the target genes for ZNF300, we sought to determine its DNA binding sequence in vitro. A random oligonucleotide selection strategy was used to identify the consensus DNA sequence recognized by ZNF300. Then, mutational electrophoretic mobility shift assays were used to confirm the stringency of the selected DNA consensus for ZNF300. Furthermore, we investigated the DNA-binding property and transcriptional function of ZNF300 using the human IL-2R $\beta$ promoter, which contains a ZNF300-binding site. IL-2R $\beta$, also named CD122, is a critical signaling component of the IL-2/IL-2Rs system, and is required for a normal immune response [5-8]. We demonstrated that ZNF300 recognizes a novel DNA binding site, $\mathrm{C}(\mathrm{t} / \mathrm{a}) \mathrm{GGGGG}(\mathrm{c} / \mathrm{g}) \mathrm{G}$, and activates the IL-2R $\beta$ promoter.

\section{MATERIALS AND METHODS}

\section{Plasmid construction}

The GST-ZF300 expression plasmid pGEX-ZF300 was generated by PCR amplifying the 12 zinc fingers of ZNF300 with the sense primer 5'-CTGGATCCGAAATACACAATCCCTT-3' and the antisense primer 5'-TACCTCGAGTTTCTGTGGCCAGTT-3', followed by cloning the product in frame with the glutathione S-transferase (GST) coding sequence into the Bam H I/Xho I-digested pGEX-5x-2 vector (Phamacia Biotech). The sense primer 5'ATGAAGCTTGCCACCATGATGAAGTCCCAGGGG3' and the 
antisense primer 5'-GCCGGATCCTTATGATTTTACC-3' were used to amplify the ZNF300 open reading frame in the pEGFP/ZNF300 plasmid (from +268 to +2080 relative to the transcription start site), and the PCR product was digested with $B a m \mathrm{H} \mathrm{I} / H i n d$ III, and then inserted into the BamH I/Hind IIIdigested pcDNA3.0 vector to construct the plasmid pcDNA-ZNF300 [4]. Human genomic DNA was extracted from normal human fresh blood, and used as the template to amplify the IL-2R $\beta$ promoter region (nt $+97 \sim-582$ ) with the sense primer IL-2Ru 5'-CCGGCTAGCGCCTGTATCAAAATAGCTCATGTGC-3' and the antisense primer IL-2Rd 5'-CGCAAGCTTTGGCTGAGACATGG GGCGGTGG-3'. The PCR product of the IL-2R $\beta$ promoter was digested with Nhe I/Hind III and then inserted into the Nhe I/Hind III-digested pGL3 basic vector to construct the pGL3-IL-2R $\beta$ vector. Overlap extension PCR mutagenesis was used to introduce mutations (underlined) into the ZNF300 binding site in the IL-2R $\beta$ promoter region with the primers IL-2Ru, IL-2Rd, IL-2RM1 (5'GCCTCCTACGCTGGGATAGGCACACACACACAC3') and IL-2RM2 (5'GTGTGTGTGTGTGCCTATCCCAGCGTAGGAGGC3'), using the pGL3-IL-2R $\beta$ plasmid as the template. In brief, a 440-bp segment was amplified using the primers IL-2Ru and IL-2RM1. In a separate reaction, a 270-bp segment was amplified using the primers IL-2Rd and IL-2RM2. Subsequently, the two PCR products were purified and combined in a second round of PCR with the primer pair IL-2Ru/IL-2Rd. A 697-bp PCR product was cloned into the Nhe I/Hind III-digested pGL3 basic vector to construct pGL3-IL-2R $\beta$ mut. The correct orientation and sequences of all the constructs were confirmed by DNA sequencing.

\section{The expression and purification of the GST-ZF300 fusion protein and GST}

The pGEX-ZF300 plasmids were transformed into E. coli BL21 (DE3). After reaching a density of $0.6 \mathrm{OD}$ (optical density) at $600 \mathrm{~nm}$, cells were induced with $1 \mathrm{mM}$ IPTG at $25^{\circ} \mathrm{C}$ for 5 hours to produce the GST-ZF300 fusion protein. Then the cells were collected and resuspended with ice-cold 1X PBS buffer plus $0.5 \mathrm{mM}$ dithiothreitol (DTT). The resuspended cells were ultra-sonicated and spun. The soluble fraction was applied to a glutathione Sepharose column preequilibrated with $1 \mathrm{X}$ PBS. The column was washed in the same buffer and the bound proteins were eluted in $30 \mathrm{mg} / \mathrm{ml} \mathrm{pH} 8.0$ reduced glutathione (GSH). The elution was further dialyzed in ice-cold PBS buffer and $0.5 \mathrm{mM}$ DTT, and then concentrated using 10,000 MWCO Millipore (Millipore China Ltd.). The purified GST-ZF300 fusion protein was stored at $-80^{\circ} \mathrm{C}$. The GST protein was purified similarly.

\section{The random oligonucleotide selection assay}

The random oligonucleotide selection assay was performed essentially as described by Pierrou and Zweidler-McKay [9, 10]. A random library of DNA sequences was generated by synthesizing Ts oligonucleotides containing a 25-bp random sequence flanked on each side by 17 bp $\left(5^{\prime}\right.$ CCTCACTTCGGTC $(\mathrm{N})_{25}$ 
CAGTG ACTCCGAAGCTT-3'). One overlapping, complementary oligonucleotide TsB (5'-AAGCTTCGGAGTCACTG-3') was annealed and extended by Klenow fragment (Takara) to yield a mixture of double-stranded DNA fragments. This mixture was incubated for $30 \mathrm{~min}$ with purified GST-ZF300 fusion protein bound to the glutathione-agarose beads in a binding buffer consisting of $25 \mathrm{mM} \mathrm{N}-2$ hydroxyethylpiperazine- $N 9$-2-ethanesulfonic acid HEPES (pH 7.5), $70 \mathrm{mM} \mathrm{KCl}, 0.1 \mathrm{mM} \mathrm{ZnCl}_{2}, 10 \mathrm{mM} \mathrm{MgCl}_{2}, 0.1 \%$ Nonidet P-40, $1 \mathrm{mM}$ dithiothreitol (DTT), $5 \%$ glycerol, and $0.2 \mathrm{mg}$ of acetylated bovine serum albumin (BSA, New England Biolabs). The beads were centrifuged and washed ten times with binding buffer and twice with $\mathrm{ddH}_{2} \mathrm{O}$, then boiled for $5 \mathrm{~min}$ in $50 \mu \mathrm{l}$ $\mathrm{ddH}_{2} \mathrm{O}$. Half of the oligonucleotide mixture eluted from the beads was used for a 30-cycle PCR amplification with the primers TsA (5'-GATCCTCACTTCG GTC-3') and TsB. The PCR products were used for the next cycle of the DNAprotein binding selection. The cycle was repeated 4 more times, but with the number of cycles in the PCR reactions changed in each subsequent round to 25 , 20,20 , and 30, respectively. After five rounds of selection, the products were cloned into pGEM-T easy vectors, and 19 individual $E$. coli transformants were isolated and sequenced.

\section{Electrophoretic mobility shift assays (EMSAs)}

To test the DNA binding properties of ZNF300, probes were generated by PCR amplification of the random DNA library with the primers TsA and TsB, and then the products were digested with $B a m \mathrm{H}$ I, and terminally $\alpha-{ }^{32}$ p labeled using a Klenow fragment (Takara). About $0.4 \mathrm{nmol}$ probes were incubated at $20^{\circ} \mathrm{C}$ for 35 minutes with $600 \mathrm{ng}$ of purified GST-ZF300 or GST proteins in the shift buffer, consisting of $10 \mathrm{mM}$ Hepes $\left(\mathrm{K}^{+} \mathrm{pH} 8.0\right), 0.06 \mathrm{M} \mathrm{KCl}, 4 \mathrm{mM} \mathrm{MgCl} 2,0.1 \mathrm{mM}$ EDTA, $0.25 \mathrm{mM}$ DTT, $0.1 \mathrm{mg} / \mathrm{ml} \mathrm{BSA}, 0.25 \mathrm{mM} \mathrm{DTT}, 0.1 \mathrm{mM} \mathrm{ZnCl} 2$, and $10 \%$ glycerol. Samples were loaded onto $5 \%$ non-denaturing polyacrylamide gel (50 mM Tris, $50 \mathrm{mM}$ glycine, $29: 1$ acrylamide/bisacrylamide $(25.3 \%$, v/v), $0.1 \mathrm{mM}$ $\mathrm{ZnCl}_{2}$, 5\% glycerol, $0.1 \%$ APS, $0.01 \%$ TEMED) and electrophoresed in $50 \mathrm{mM}$ Tris-glycine buffer. We chose the Tris-glycine buffer system (pH 9.2) for electrophoresis because the isoelectric point of the GST-ZF300 protein is relatively high $(\mathrm{p} I=8.9)$. The gel was analyzed by autoradiography.

The oligonucleotide T-clone 5 probe was generated as follows. The sense primer (5'-GAGTCACTGGGGGCGTGGCTC-3') and the antisense primer (5'-GAGCCACGCCCCCAGTGACTC-3') were annealed in $10 \mathrm{mM}$ Tris- $\mathrm{HCl}$, $100 \mathrm{mM} \mathrm{NaCl}, 1 \mathrm{mM}$ EDTA buffer $(\mathrm{pH} \mathrm{8.0)}$ and end-labeled with 2500 $\mathrm{Ci} / \mathrm{mmol} \gamma_{-}^{32} \mathrm{P}$ ATP using T4 polynucleotide kinase (Promega). The unincorporated $\gamma_{-}{ }^{32} \mathrm{P}$ ATP was removed by passage through a G-25 column (Amersham Biosciences). To generate the wild-type IL-2R $\beta$ probe (from -177 to -145), the sense primer (5'-GTGTGTGTGTGTGCCGCCCCCAGCGTAGGA GGC-3') and antisense primer (5'-GCCTCCTACGCTGGGGGCGGCACACAC ACACAC-3') were annealed and end-labeled similarly. $35 \mathrm{fmol}$ of the indicated specific $\gamma^{32} \mathrm{P}$ labeled probes were incubated at $20^{\circ} \mathrm{C}$ for $35 \mathrm{~min}$ with $300 \mathrm{ng}$ of 
purified GST-ZF300 proteins in the shift buffer. Samples were loaded onto $5 \%$ non-denaturing polyacrylamide gel and electrophoresed in $50 \mathrm{mM}$ Trisglycine buffer. The gel was analyzed by autoradiography. For the competition assay, unlabeled oligonucleotides were synthesized with base substitution at the consensus-binding site as indicated, and a 100-, 200-, 300- or 500-fold molar excess of the indicated unlabeled oligonucleotides was added to the binding reactions just before the addition of the radiolabeled probes.

In the supershift experiments, the Jurkat nuclear extracts were prepared as described previously [11]. $35 \mathrm{fmol}$ of the labeled T-clone 5 probe was incubated with $20 \mu \mathrm{g}$ of nuclear extracts in $1 \mathrm{X}$ Gel Shift Binding Buffer $(4 \%$ glycerol, $1 \mathrm{mM}$ $\mathrm{MgCl}_{2}, 0.5 \mathrm{mM}$ EDTA, $0.5 \mathrm{mM}$ dithiothreitol, $50 \mathrm{mM} \mathrm{NaCl}, 10 \mathrm{mM}$ Tris- $\mathrm{HCl}$, $\mathrm{pH} 7.5$, and $0.05 \mathrm{mg} / \mathrm{ml}$ poly ( $\mathrm{dI}-\mathrm{dC})$ ) for $30 \mathrm{~min}$ at room temperature. Then, anti-ZNF300 antibody (generated by Beijing Biosynthesis Biotechnology, China) or pre-immune rabbit serum (Zhongshan, Biotechnology, China) was added to the binding reaction, followed by incubation for 1 hour at room temperature. Samples were then electrophoresed on 5\% non-denaturing polyacrylamide gels $(25 \mathrm{mM}$ Tris, $190 \mathrm{mM}$ Glysine, $1 \mathrm{mM}$ EDTA, 29:1 acrylamide/bisacrylamide $(25.3 \%, \mathrm{v} / \mathrm{v}), 5 \%$ glycerol $)$, and the gel was analyzed by autoradiography.

\section{Cell culture, transient transfection and dual luciferase assay}

The mammalian cell lines HeLa and 293T were cultured in DMEM (Dulbecco's modified Eagle's medium) with 10\% Bovine Calf Serum (GIBCO, Invitrogen Corporation, USA). The Jurkat cell line was cultured in RPMI1640 medium supplemented with $10 \%$ fetal calf serum.

The Sofast ${ }^{\mathrm{TM}}$ Transfection system (Xiamen Sunma Biotechnology Co., Ltd) was used for transient transfection. This was done with the indicated $100 \mathrm{ng}$ firefly luciferase reporter construct and the internal control Renilla luciferase reporter construct, pRL-TK (Promega; the firefly luciferase reporter construct and pRL-TK were in a ratio of 10:1), which contains the Renilla luciferase gene driven by the herpes simplex virus thymidine kinase (TK) promoter. Co-transfection experiments were performed on HeLa cells by adding $500 \mathrm{ng}$ of the pcDNA3.0 vector or pcDNA-ZNF300 expression vector together with the respective luciferase reporter construct and the internal control plasmid pRL-TK (Promega). 48 hours after transfection, cells were harvested, lysed and assayed for luciferase activity according to the protocol from Promega. Triplicate samples were measured for each construct, and the average values of the ratio of firefly luciferase light units to Renilla luciferase light units were used for data analysis. The results show the mean values of three independent experiments with standard error.

\section{Western blot assay}

Protein samples $(100 \mu \mathrm{g})$ were analyzed on $10 \%$ SDS-polyacrylamide gels and electrophoretically transferred onto nitrocellulose membranes. The membranes 
CELL. MOL. BIOL. LETT.

were blocked in 5\% non-fat dry milk for 2 hours, and probed with the antiZNF300 antibody. Following incubation with the HRP-conjugated secondary antibody (Pierce), proteins were detected using the ECL reagent (Pierce).

\section{RESULTS AND DISCUSSION}

In vitro isolation of oligonucleotides containing a ZNF300 binding site through the random oligonucleotide selection strategy

The $\mathrm{C}_{2} \mathrm{H}_{2}$-type zinc finger proteins usually function as transcription factors and bind to DNA, RNA and RNA-DNA hybrids, but mostly interact with DNA [12-14]. ZNF300 is a nuclear protein [4], and thus may be a sequence-specific DNAbinding protein. To investigate this hypothesis, the twelve zinc finger motifs of ZNF300 were fused with GST to produce GST-ZF300 protein $(64 \mathrm{kDa}, \mathrm{p} I=8.93)$. Then, the EMSA assay was performed with the PCR-based $\alpha-{ }^{32}$ p labeled DNA random library (Fig. 1A) and purified with GST-ZF300 or GST. Unlike GST, the GST-ZF300 fusion protein retarded the electrophoresis of the probes, suggesting that the zinc fingers of ZNF300 function as a DNA-binding domain (Fig. 1B).

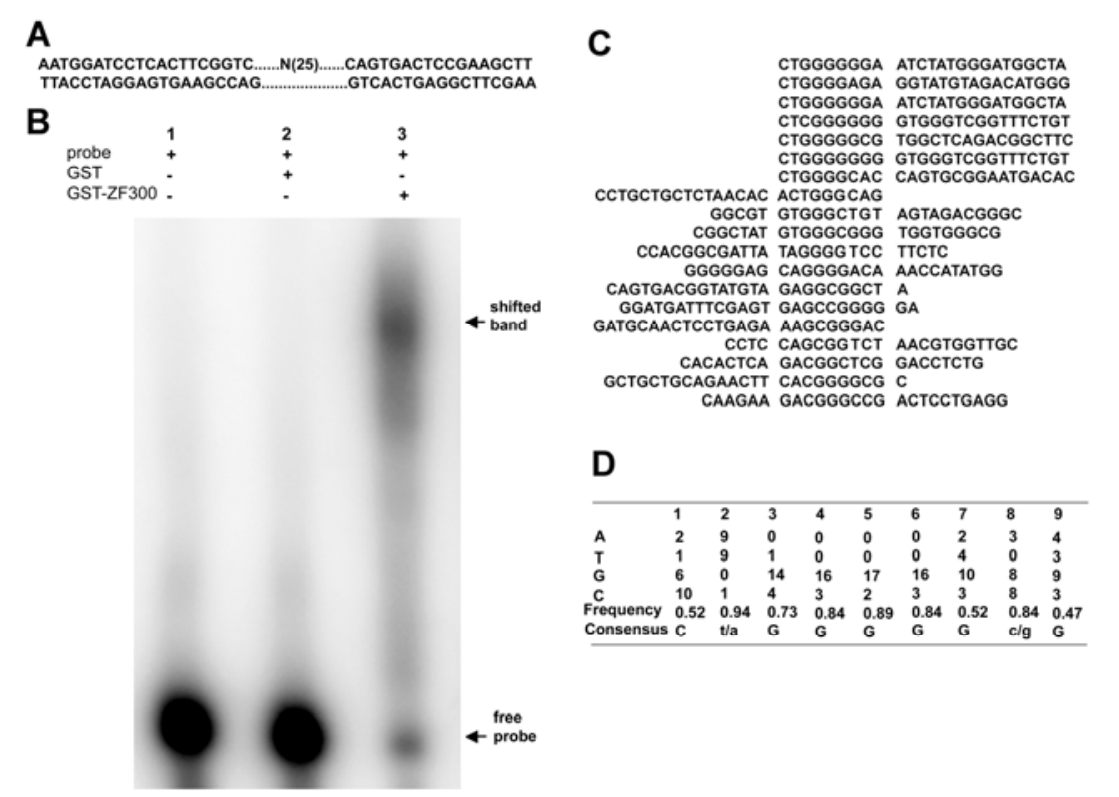

Fig. 1. In vitro isolation of oligonucleotides containing ZNF300 binding sites. A - The random oligonucleotide library used in the DNA-binding site selection assay. B - An $\alpha-{ }^{32} \mathrm{p}$ labeled PCR-amplified random oligonucleotide library was used as the probe for EMSA without any protein, with purified GST protein or with purified GST-ZF300. C - The sequences of the 19 affinity-selected oligonucleotides bound by ZNF300. D - The nucleotide frequencies of the 19 selected oligonucleotides and the consensus ZNF300 binding site. 
To determine the consensus ZNF300 DNA binding site, we used the random oligonucleotide selection strategy as described in the materials and methods section. The products after five rounds of selection were cloned into pGEM-T easy vectors, and 19 independent clones were sequenced and analyzed. As shown in Fig. 1C and D, the alignment of these oligonucleotides allowed us to deduce the consensus ZNF300 DNA binding site of $\mathrm{C}(\mathrm{t} / \mathrm{a}) \mathrm{GGGGG}(\mathrm{g} / \mathrm{c}) \mathrm{G}$. Database comparisons revealed that the consensus ZNF300 DNA binding site did not contain a binding site for other previously characterized transcription factors, and thus represented a novel DNA binding site.

\section{Characterization of the binding specificity of the selected ZNF300 binding site for ZNF300}

A relative binding affinity analysis of the cloned ZNF300 binding sites for ZNF300 revealed that the insert sequence from T-clone 5, which contained the consensus CTGGGGGCG, showed the highest binding affinity for ZNF300 (data not shown). Thus, the insert sequence from T-clone 5 was used as a probe for further analysis.

We examined the specificity of the probe binding to ZNF300 by performing competition assays. As shown in Fig. 2A, the incubation of GST-ZF300 with the probe alone without any competitors resulted in the formation of a shifted band (Fig. 2A, lane 2). A 200-fold molar of the unlabeled probe was able to eliminate most of the shifted band (Fig. 2A, lane 3), while a molar ratio as high as 500-fold of the unlabeled AT-rich DNA, known as the target for the transcription factor OCT1, failed to compete away the DNA-protein complex (Fig. 2A, lane 7) [15]. These results indicate that the sequence selected by the random oligonucleotide selection strategy binds to ZNF300 in a highly specific manner.

Next, competition EMSA assays were performed with mutagenized binding sites to further characterize the binding specificity. A series of mutations were introduced into the consensus (CTGGGGGCG). First, triplet nucleotides in the consensus were substituted individually for competitive EMSA. As shown in Fig. 2B, the presence of a 200-fold molar of wild-type unlabeled oligonucleotides in the reaction competed efficiently against the formation of the complex (Fig. 2B, lane 2 versus lane 3). By contrast, the DNA-protein complex was not affected by the mutant oligonucleotides mut1, mut2 and mut3 (Fig. 2B, lanes 4-6). Thus, all of the three triplet nucleotides in the consensus are required for the maximal binding affinity for ZNF300.

We then examined whether two base-pair substitutes in the consensus will affect the binding affinity for ZNF300. As shown in Fig. 2C, the unlabeled wild-type probe competed effectively against the formation of the complex, as did the unlabeled mutant oligonucleotide mt1 (Fig. 2C, lanes 3 and 4). By contrast, the unlabeled mutant oligonucleotides $\mathrm{mt} 2, \mathrm{mt} 3$ and $\mathrm{mt} 4$ failed to prevent the complex formation (Fig. 2C, lanes 5-7). Thus, the sequence GGGGGCG is the minimal essential binding site required for ZNF300. 
A

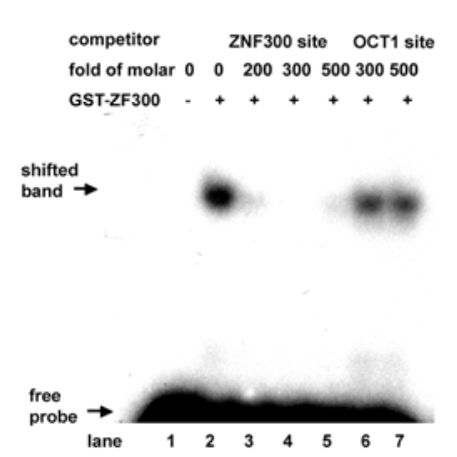

B

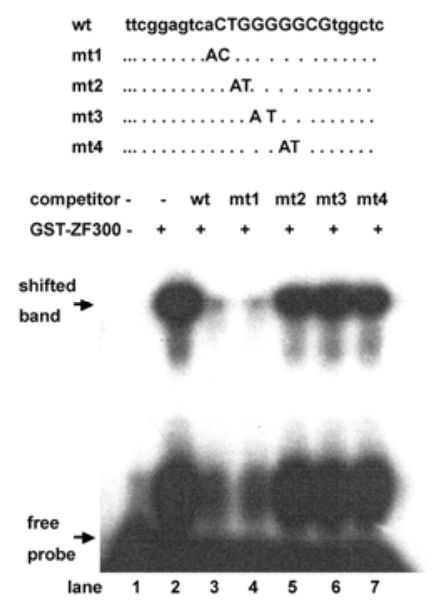

C
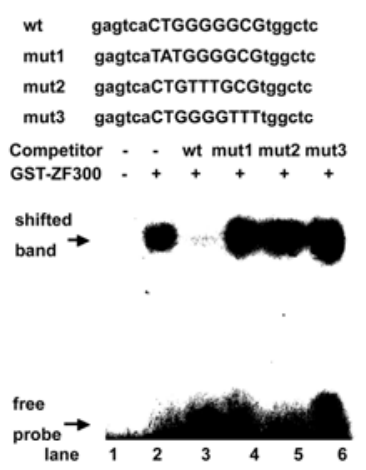

D wt

ttcggagtcaCTGGGGGCGtggctc

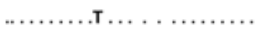

.......................

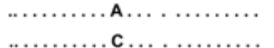

(

$T \ldots \ldots \ldots$

T. .

........... T

$T$

............. т.......

.....................

....................

.....................

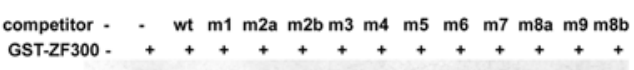

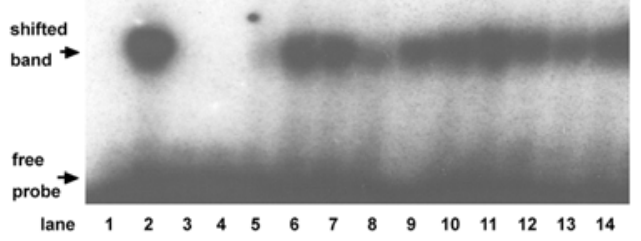

Fig. 2. Mutations in the ZNF300 binding site in the T-clone 5 reduce its affinity for ZNF300. The labeled T-clone 5 probe was incubated with purified GST-ZF300 protein in the presence of the indicated unlabeled competitors. The consensus nucleotides involved in binding are shown in capital letters. In the mutants, the mutated bases are shown below with the unchanged bases represented by dots. A - Competition EMSA showed specific binding of ZNF300 to the ZNF300 binding site. The wild-type probe and the probe containing the OCT-1 binding site were used for unlabeled competitors to show the specificity of the binding. B, C, D - Mutational analysis of the ZNF300 binding site. 200 -fold molar excesses of various unlabeled wild-type or mutant oligonucleotide competitors were added to the reaction for EMSA. Note that most mutations reduced the ability of the oligonucleotide to compete for binding to ZNF300 (see text for more details).

Finally, we sought to clarify whether each of the seven bases identified in Fig. 2C was absolutely required for ZNF300 binding. A series of mutant oligonucleotides with single base-pair substitutions were synthesized and 
analyzed in the competition assay. As shown in Fig. 2D, M1 (change C to T) and M2a (change $\mathrm{T}$ to $\mathrm{A}$ ) had almost the same competing ability as the unlabeled wild type oligonucleotide (Fig. 2D, lanes 3-5), while M2b (change $\mathrm{T}$ to $\mathrm{C}$ ) failed to abolish the DNA-protein complex formation (Fig. 2D, lane 6). The unlabeled mutant oligonucleotide M4 competed to some extent, while mutant oligonucleotides M3 and M5 to M9 had little effect on the DNA-protein complex (Fig. 2D lanes 6, 7 and 9-13). Although the frequency for $G$ is the same as $\mathrm{C}$ at the eighth position from the library screen, $\mathrm{M} 8 \mathrm{~b}$ (change $\mathrm{C}$ to $\mathrm{G}$ ) failed to compete against the DNA-protein complex in the context of this particular binding site (Fig. 2D, lane 14). In conclusion, the sequence GGGGGCG contained the minimal essential binding site required for ZNF300. However, the first dinucleotide $\mathrm{CT}$ in the nine nucleotide consensus also participated in the binding to ZNF300, because a mutation at this position to $\mathrm{CC}$ affected binding to some extent, whereas changing CT to AC (Fig. 2C, lane 4), TT or CA (Fig. 2D, lanes 4 and 5) had little effect on the binding activity.

\section{Endogenous ZNF300 protein also binds the ZNF300 binding site}

ZNF300 encodes a predicted protein with a molecular weight $(\mathrm{Mr})$ of $68.7 \mathrm{kDa}$. To study the DNA binding property of endogenous ZNF300 protein, a polyclonal antibody against human ZNF300 was prepared. As shown in Fig. 3A, a single band of the expected size (about $68 \mathrm{kDa}$ ) was recognized by the antibody in $293 \mathrm{~T}$ cells, Jurkat cells and HeLa cells in the Western blot assay, demonstrating the specificity of this antibody to ZNF300.

To confirm that the endogenous ZNF300 protein and GST-ZF300 expressed in $E$. coli have identical DNA-binding characteristics, the probe containing the ZNF300 binding site was incubated with Jurkat nuclear extracts, which express endogenous ZNF300, and the mixture was subjected to super-shift assays with the anti-ZNF300 antibody or pre-immune serum. A DNA-protein complex formed when the probe was incubated with Jurkat nuclear extracts (Fig. 3B, lane 2). The addition of anti-ZNF300 antibody but not pre-immune serum to the binding reaction resulted in the appearance of a retarded band (Fig. 3B, lane 3 versus lanes 2 and 4). These results revealed the presence of ZNF300 in the DNA-protein complex, and the endogenous ZNF300 protein could recognize the consensus DNA sequence selected by the GST-ZF300 expressed in E. coli.

The DNA binding specificity of the endogenous ZNF300 was investigated in competition assays. The DNA-protein complex was competed with efficiently by the unlabeled wild-type oligonucleotide wt but not by the mutant oligonucleotides mut2 and mut3 (Fig. 3B, lane 5 versus lanes 7 and 8). Surprisingly, the unlabeled mutant oligonucleotide mut 1 also eliminated most of the DNA-protein complex (Fig 3B, lane 6), although it had little effect on the binding by GST-ZF300. These results suggested endogenous full-length ZNF300 but not purified GST-ZF300 could bind to mut1. Regardless, endogenous ZNF300 can bind to the selected ZNF300 binding site in a specific manner. 

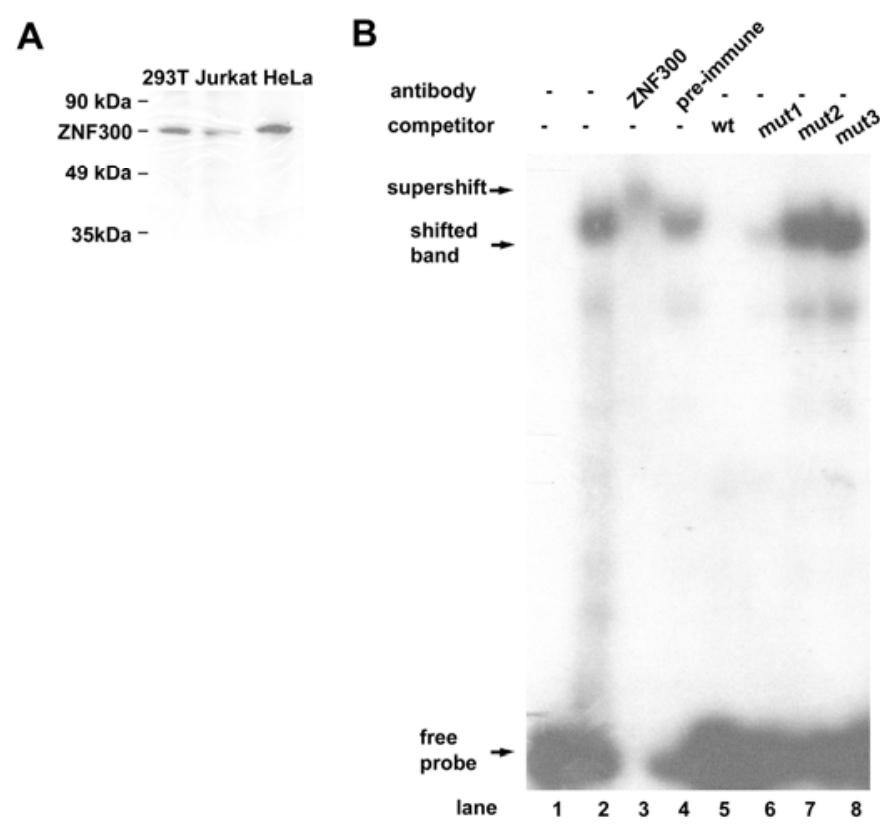

Fig. 3. The ZNF300 gene encodes a $68-\mathrm{kDa}$ protein that binds to the selected ZNF300 binding site. A - Western blot analysis of ZNF300 expression in 293T, Jurkat and HeLa cells. Note that the anti-ZNF300 antibody recognizes a single band of the expected size (about $68 \mathrm{kDa}$ ) in the 293T, Jurkat and HeLa cells. B - Endogenous ZNF300 binds to the selected ZNF300 binding site. EMSAs were performed with the T-clone 5 probe and Jurkat nuclear extracts. Binding specificity was determined using wild type or mutant oligonucleotides (see the sequence in Fig. 2A) as competitors. The identity of the ZNF300 complex was confirmed by the supershift assay using the anti-ZNF300 antibody but not the pre-immune serum (PI). The supershift band is indicated with an arrow.

\section{Overexpression of ZNF300 enhances IL-2R $\beta$ promoter activity}

To identify potential target promoters of ZNF300, we searched the Eukaryotic Promoter Database with the newly identified ZNF300 binding sites, and discovered that many eukaryotic promoters contained potential ZNF300 binding sites. As described above, ZNF300 showed the highest binding affinity to the DNA consensus CTGGGGGCG in vitro, so we focused on the promoters that contained this DNA consensus.

Of the selected promoters, the IL-2R $\beta$ promoter region contains one potential ZNF300 binding site near the transcription initiation site (Fig. 4A) [16]. When EMSA was performed with the IL-2R $\beta$ probe containing the ZNF300 binding site (from -177 to -145 ) and purified GST-ZF300 protein, a shifted band was detected (Fig. 4B, lane 1). The shifted band was abolished by adding the unlabeled wild-type (wt) but not the mutant oligonucleotide containing mutant nucleotides in the ZNF300 binding site (mut) (Fig. 4B, lane 3 versus lane 2). Thus, ZNF300 binds to the ZNF300 binding site in the IL-2R $\beta$ promoter region. 
To further examine the transcriptional regulatory function of ZNF300, we focused our attention on the IL-2R $\beta$ promoter and investigated the effect of ZNF300 on this promoter. The luciferase reporter plasmid construct pGL3-IL-2R $\beta$ or the mutation construct pGL3-IL-2R $\beta$ mut could be transiently co-transfected into the HeLa cells with the expression plasmid pcDNA-ZNF300 or the empty vector pcDNA3.0. As shown in Fig. 4C, co-transfection of the expression plasmid with pcDNA3-ZNF300 could result in an obvious increase in luciferase activity, while mutation of the ZNF300 binding site in the promoter region greatly diminished the luciferase activity enhanced by ZNF300. Thus, ZNF300 can activate the IL-2R $\beta$ promoter activity, and the ZNF300 binding site in the IL-2R $\beta$ promoter region is required for the promoter activity activated by ZNF300.

A

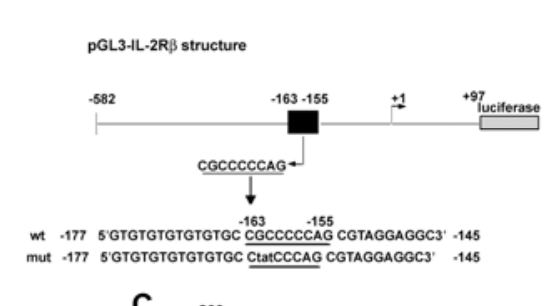

B

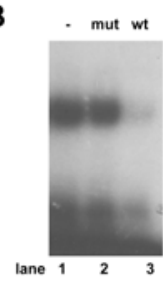

C

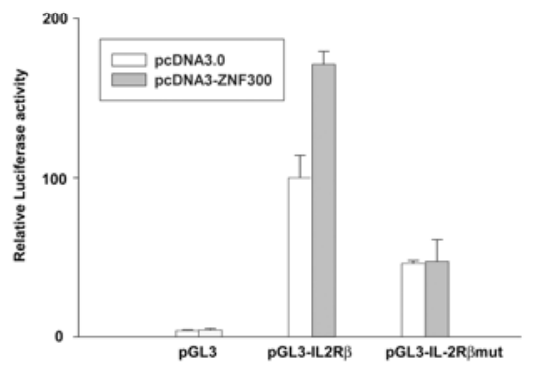

Fig. 4. ZNF300 binds to the ZNF300 binding site in the IL-2R $\beta$ promoter region and activates the IL-2R $\beta$ promoter. A - Schematic diagram of the reporter construct containing the wild-type or mutant ZNF300 binding site in the IL-2R $\beta$ promoter region. The DNA sequences containing the potential wild-type and mutant ZNF300 binding site are indicated (underlined). B - The mutational EMSA assay indicated that the ZNF300 binding site (underlined) in the IL-2R $\beta$ promoter region was involved in ZNF300 binding. Purified GST-ZF300 protein and the IL-2R $\beta$ probe were used in the EMSA assay. The wild-type probe (wt) and mutant probe (mut) were used for the unlabeled competitors to show the specificity of the binding. $\mathrm{C}-\mathrm{HeLa}$ cells were transiently co-transfected with the pGL3IL-2R $\beta$ construct, pGL3-IL-2R $\beta$ mut or promoter-less pGL3 reporter plasmid and the expression plasmid pcDNA3-ZNF300 or empty pcDNA3.0 vector (as a control). Note that ZNF300 enhanced the wild-type but not mutant IL-2R $\beta$ promoter activity. As an internal control, the pRL-TK plasmid was used to normalize the transfection efficiency. The results shown are the mean values of three independent experiments with standard errors. 
ZNF300 is a typical KRAB-containing $\mathrm{C}_{2} \mathrm{H}_{2}$ zinc finger protein. The KRAB domain exhibits transcription repressor activity [4]. However, in our study, the full-length ZNF300 functions not as a repressor but as an activator on the IL-2R $\beta$ promoter. Thus, our findings are consistent with a growing body of evidence showing that KRAB-containing zinc finger proteins can function as transcription activators, although the detailed mechanism remains unclear. One possibility is that $\mathrm{KRAB}$-containing zinc finger proteins recruit other co-factors to function as transcriptional activators. For example, K-RBP can interact with the cellular RTA cofactor to activate viral gene expression [17]. Another possibility is that another domain of the KRAB-box-containing zinc finger protein functions as the activator domain. For example, ZNF641 can activate the transcriptional activity of AP-1 and SRE, and the linker between KRAB-box and zinc finger motifs represents the basal activation domain [18]. How ZNF300 functions as an activator in vivo will be an important issue to address in the future.

In summary, we successfully identified the consensus DNA binding sequence of ZNF300, and identified many putative target genes of ZNF300 through a bioinformatics search with the sequence. We further demonstrated that ZNF300 could activate one of its newly identified targets, the IL-2R $\beta$ promoter. Further studies on how ZNF300 regulates this and other targets, especially in vivo, should provide critical insights on the biological functions of this higher mammal-specific gene.

Acknowledgements. This study was supported by the National High Technology Research and Development Program of China (grant No. 2006AA02A306), the National Natural Science Foundation of China (grant No. 30500266) and the Program of Introducing Talents of Discipline to Universities (grant B06018).

\section{REFERENCES}

1. Schuh, R., Aicher, W., Gaul, U., Cote, S., Preiss, A., Maier, D., Seifert, E., Nauber, U., Schroder, C. and Kemler, R. A conserved family of nuclear proteins containing structural elements of the finger protein encoded by Kruppel, a Drosophila segmentation gene. Cell $\underline{47}$ (1986) 1025-1032.

2. Dai, K.S. and Liew, C.C. Chromosomal, in silico and in vitro expression analysis of cardiovascular-based genes encoding zinc finger proteins. J. Mol. Cell. Cardiol. 31 (1999) 1749-1769.

3. Decker, E.L., Nehmann, N., Kampen, E., Eibel, H., Zipfel, P.F. and Skerka, C. Early growth response proteins (EGR) and nuclear factors of activated T cells (NFAT) form heterodimers and regulate proinflammatory cytokine gene expression. Nucleic Acids Res. 31 (2003) 911-921.

4. Gou, D.M., Wang, J., Gao, L., Sun, Y., Peng, X., Huang, J. and Li, W.X. Identification and functional analysis of a novel human $\mathrm{KRAB} / \mathrm{C}_{2} \mathrm{H}_{2}$ zinc finger gene ZNF300. Biochim. Biophys. Acta 1676 (2004) 203-209. 
5. Sharon, M., Gnarra, J.R. and Leonard, W.J. A 100-kilodalton protein is associated with the murine interleukin 2 receptor: biochemical evidence that p100 is distinct from the alpha and beta chains. Proc. Natl. Acad. Sci. USA $\underline{87}$ (1990) 4869-4873.

6. Suzuki, H., Duncan, G.S., Takimoto, H. and Mak, T.W. Abnormal development of intestinal intraepithelial lymphocytes and peripheral natural killer cells in mice lacking the IL-2 receptor beta chain. J. Exp. Med. 185 (1997) 499-505.

7. Suzuki, H., Kundig, T.M., Furlonger, C., Wakeham, A., Timms, E., Matsuyama, T., Schmits, R., Simard, J.J., Ohashi, P.S., Griesser, H. Deregulated T-cell activation and autoimmunity in mice lacking interleukin-2 receptor beta. Science $\underline{268}$ (1995) 1472-1476.

8. Espinoza-Delgado, I., Ortaldo, J.R., Winkler-Pickett, R., Sugamura, K., Varesio, L. and Longo, D.L. Expression and role of p75 interleukin 2 receptor on human monocytes. J. Exp. Med. 171 (1990) 1821-1826.

9. Pierrou, S., Enerback, S. and Carlsson, P. Selection of high-affinity binding sites for sequence-specific, DNA binding proteins from random sequence oligonucleotides. Anal. Biochem. 229 (1995) 99-105.

10. Zweidler-Mckay, P.A., Grimes, H.L., Flubacher, M.M. and Tsichlis, P.N. Gfi-1 encodes a nuclear zinc finger protein that binds DNA and functions as a transcriptional repressor. Mol. Cell. Biol. 16 (1996) 4024-4034.

11. Kim, J.C., Yoon, J.B., Koo, H.S. and Chung, I.K. Cloning and characterization of the 5'-flanking region for the human topoisomerase III gene. J. Biol. Chem. 273 (1998) 26130-26137.

12. Wolfe, S.A., Nekludova, L. and Pabo, C.O. DNA recognition by Cys2His2 zinc finger proteins. Annu. Rev. Biophys. Biomol. Struct. 29 (2000) 183-212.

13. Mendez-Vidal, C., Wilhelm, M.T., Hellborg, F., Qian, W. and Wiman, K.G. The p53-induced mouse zinc finger protein wig-1 binds double-stranded RNA with high affinity. Nucleic Acids Res. 30 (2002) 1991-1996.

14. Finerty, P.J., Jr. and Bass, B.L. A Xenopus zinc finger protein that specifically binds dsRNA and RNA-DNA hybrids. J. Mol. Biol. 271 (1997) 195-208.

15. O'Neill, E.A., Fletcher, C., Burrow, C.R., Heintz, N., Roeder, R.G. and Kelly, T.J. Transcription factor OTF-1 is functionally identical to the DNA replication factor NF-III. Science 241 (1988) 1210-1213.

16. Lin, J.X., Bhat, N.K., John, S., Queale, W.S. and Leonard, W.J. Characterization of the human interleukin-2 receptor beta-chain gene promoter: regulation of promoter activity by ets gene products. Mol. Cell. Biol. 13 (1993) 6201-6210.

17. Wang, S., Liu, S., Wu, M.H., Geng, Y. and Wood, C. Identification of a cellular protein that interacts and synergizes with the RTA (ORF50) protein of Kaposi's sarcoma-associated herpesvirus in transcriptional activation. J. Virol. $\underline{75}$ (2001) 11961-11973.

18. Qi, X., Li, Y., Xiao, J., Yuan, W., Yan, Y., Wang, Y., Liang, S., Zhu, C., Chen, Y., Liu, M. and Wu, X. Activation of transcriptional activities of AP-1 and SRE by a new zinc-finger protein ZNF641. Biochem. Biophys. Res. Commun. 339 (2006) 1155-1164. 